\title{
Neural Model of Dopaminergic Control of Arm Movements in Parkinson's Disease Bradykinesia
}

\author{
Vassilis Cutsuridis \\ Computational Intelligence Laboratory, Institute of Informatics and Telecommunications, \\ National Center for Scientific Research "Demokritos", Agia Paraskevi, Athens GR-15310 \\ vcutait.demokritos.gr
}

\begin{abstract}
Patients suffering from Parkinson's disease display a number of symptoms such a resting tremor, bradykinesia, etc. Bradykinesia is the hallmark and most disabling symptom of Parkinson's disease (PD). Herein, a basal ganglia-cortico-spinal circuit for the control of voluntary arm movements in PD bradykinesia is extended by incorporating DAergic innervation of cells in the cortical and spinal components of the circuit. The resultant model simulates successfully several of the main reported effects of DA depletion on neuronal, electromyographic and movement parameters of PD bradykinesia.
\end{abstract}

\section{Introduction}

The most severe symptom of Parkinson's disease is bradykinesia (i.e. slowness of movement). It is not known what causes bradykinesia because there are many pathways from the sites of neuronal degeneration to the muscles (see Figure 1). The most important pathways are: (1) the pathway from the substantia nigra pars compacta ( $\mathrm{SNc}$ ) and the ventral tegmental area (VTA) to the striatum and from the striatum to the substantia nigra pars reticulata $(\mathrm{SNr})$ and the globus pallidus internal segment (GPi) and from there to the thalamus and the frontal cortex, (2) the pathway from the SNc and the VTA to the striatum and from the striatum to the $\mathrm{SNr}$ and the GPi and from there to the brainstem, and (3) the pathway from the SNc/VTA to cortical areas such as the supplementary motor area (SMA), the parietal cortex, and the primary motor cortex (M1), and from there to the spinal cord. [12]

The currently accepted view of what causes bradykinesia is that cortical motor centers are not activated sufficiently the basal ganglia (BG) circuits. As a result, inadequate facilitation is provided to motor cortical and spinal neuron pools and hence movements are small and weak [1]. The implication of this view is that cells in the cortex and spinal cord are functioning normally. This paper suggests otherwise.

In this paper, I integrate experimental data on the anatomy and neurophysiology of the globus pallidus internal segment [19], the cortex [10] and the spinal cord structures, as well as data on PD psychophysics $[16,17,18,20]$ to extend a neural model of basal ganglia-cortex-spinal cord interactions during movement production $[2,3,4,5,6,7,8]$. Computer simulations show that disruptions of the BG output and of the SNc's DA input to frontal and parietal cortices and spinal cord may be responsible for delayed movement initiation. The main hypothesis of the model is that 


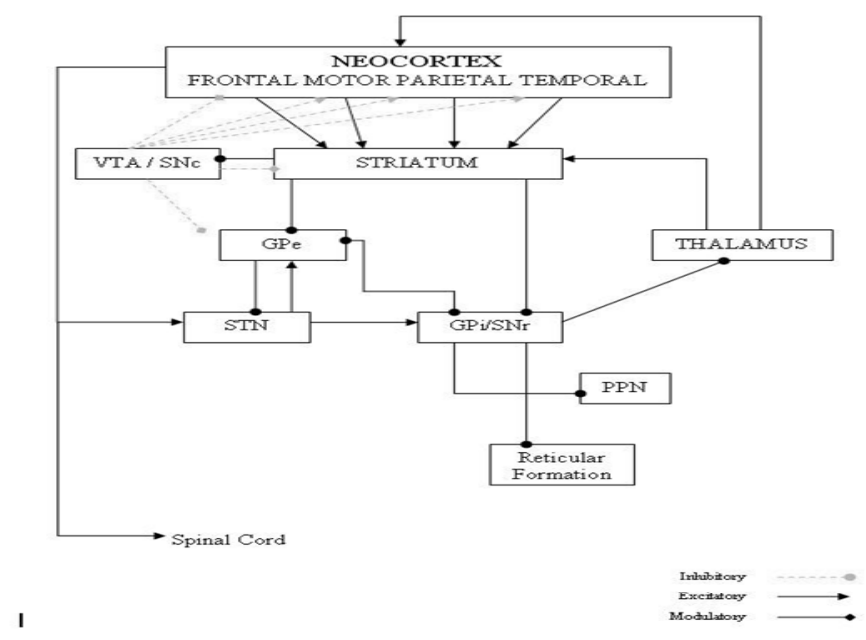

Fig. 1. Schematic diagram of dopaminergic innervation of basal ganglia and sensory-motor cortex. Arrow-ending solid lines, excitatory projections; Dot-ending solid lines, inhibitory projections; Diamond-ending dotted lines, dopamine (DA) modulatory projections; STN, subthalamic nucleus; GPi, globus pallidus internal segment; GPe, globus pallidus external segment; SNr, substantia nigra pars reticulata; SNc, substantia nigra pars compacta; VTA, ventral tegmental area; PPN, pedunculopontine nucleus.

elimination of DA modulation from the SNc disrupts, via several pathways, the buildup of the pattern of movement-related responses in the primary motor and parietal cortex, and results in a loss of directional specificity of reciprocal and bidirectional cells in the motor cortex as well as in a reduction in their activities and their rates of change. These changes result in delays in recruiting the appropriate level of muscle force sufficiently fast and in an inappropriate scaling of the dynamic muscle force to the movement parameters. A repetitive triphasic pattern of muscle activation is sometimes needed to complete the movement. All of these result in an increase of mean reaction time and a slowness of movement (i.e. bradykinesia). This work has been published in $[12,13,14,15]$.

\section{Materials and Methods}

\subsection{Basis of the Model}

Figure 2 schematizes the basal ganglio-cortico-spinal network model. As a basal ganglio-cortical network, the VITE (Vector Integration To-End point) model of [3] was chosen, which is here extended. In my proposed version of the VITE model, the types and properties of the cortically identified neurons are extended and the effects of dopamine depletion on key cortical cellular sites are studied. Briefly in the model, an arm movement difference vector (DV) is computed in parietal area 5 from a comparison of a target position vector (TPV) with a representation of the current position called perceived position vector (PPV). The DV signal then projects to area 


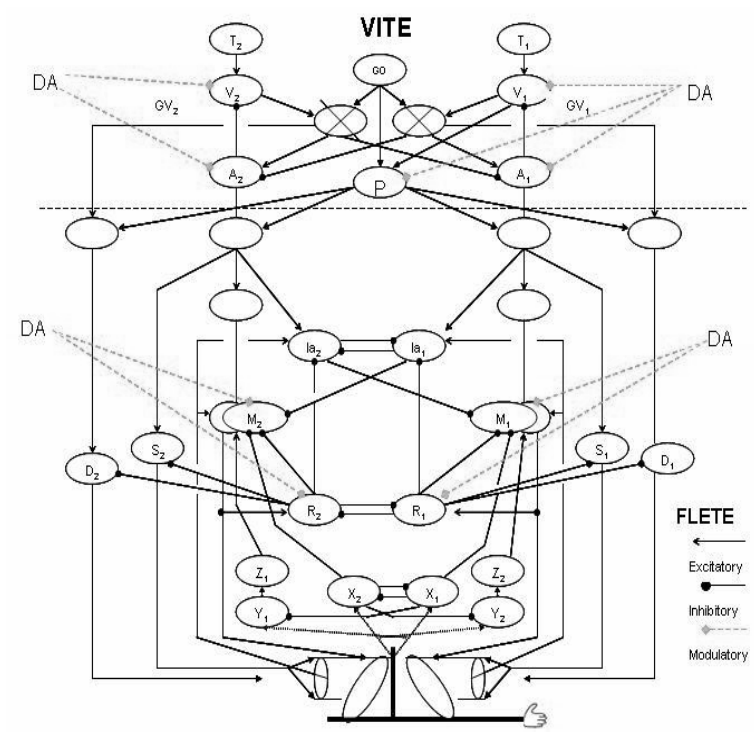

Fig. 2. Neural network representation of the cortico-spinal control system. (Top) The VITE model for variable-speed trajectory generation. (Bottom) the FLETE model of the opponent processing spinomuscular system. Arrow lines, excitatory projections; solid-dot lines, inhibitory projections; diamond dashed lines, dopamine modulatory inputs; dotted arrow lines, feedback pathways from sensors embedded in muscles; DA, dopamine modulatory signal; GO, basal ganglia output signal; $\mathrm{P}$, bi-directional co-contractive signal; $\mathrm{T}$, target position command; V, DV activity; GV, DVV activity; A, current position command; M, alpha motoneuronal $(\mathrm{MN})$ activity; R, renshaw cell activity; X, Y, Z, spinal inhibitory interneuron (IN) activities; Ia, spinal type a inhibitory IN activity; $\mathrm{S}$, static gamma $\mathrm{MN}$ activity; $\mathrm{D}$, dynamic gamma $\mathrm{MN}$ activity; 1,2, antagonist cell pair (adapted from [12]).

4, where a desired velocity vector (DVV) and a non-specific co-contractive signal (P) [9] are formed. A voluntarily scalable GO signal multiplies (i.e. gates) the DV input to both the DVV and $\mathrm{P}$ in area 4, and thus volitional-sensitive velocity and nonspecific co-contractive commands are generated, which activate the lower spinal centers. In my model, the DVV signal represents the activity of reciprocal neurons [10], and it is organized for the reciprocal activation of antagonist muscles, whereas the $\mathrm{P}$ signal represents the activity of bidirectional neurons (i.e. neurons whose activity decreases or increases for both directions of movement [10]), and it is organized for the co-contraction of antagonist muscles.

The spinal recipient of my model is the FLETE (Factorization of LEngth and Tension) model $[2,3,4,5,6]$. Briefly, the FLETE model is an opponent processing muscle control model of how spinal circuits afford independent voluntary control of joint stiffness and joint position. It incorporates second-order dynamics, which play a large role in realistic limb movements. I extended the original FLETE model by incorporating the effect of the now cortically controlled co-contractive signal (in the original FLETE model, the co-contraction signal was simply a parameter) onto its spinal elements. Finally, I studied the effects that dopamine depletion on key spinal centers has on voluntary movements. 


\subsection{Architecture}

The mathematical formalism of the basal ganglio-cortico-spinal model has been described elsewhere [12].

\section{Results}

\subsection{Dopamine Depletion Effects on the Discharge of Globus Pallidus Internal Segment Neurons}

Figure 3 shows a qualitative comparison of abnormal cellular responses of GPi neurons to striatal stimulation in MPTP-treated monkeys [19] and simulated long duration of late inhibitions (B) and oscillatory (D) GPi neuronal responses. I propose that GPi responses similar to figure $3 \mathrm{~B}$ are used to complete small amplitude

A

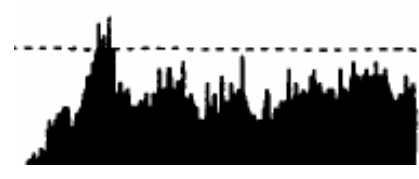

$\mathrm{C}$

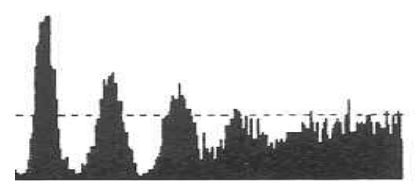

B

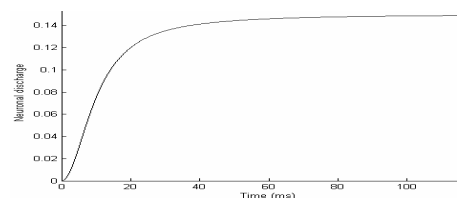

$\mathrm{D}$

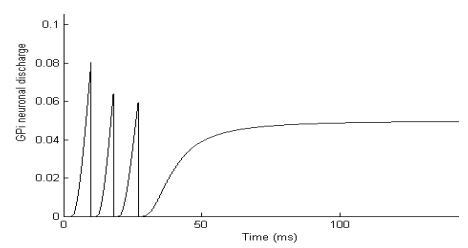

Fig. 3. Comparison of (A) peristimulus histograms (PSTH) of neuronal responses of GPi cells to striatal stimulation in MPTP-treated monkeys (adapted from Tremblay et al., 1989, Fig. 2, p. 23), (B) simulated dopamine depleted GPi neuronal response, (C) peristimulus histograms (PSTH) of abnormal oscillatory responses of GPi neurons to striatal stimulation in MPTPtreated monkeys (adapted from Tremblay et al., 1989, Fig. 2, p. 23), and (D) simulated oscillatory disrupted GPi responses. Time units in ms.

movements, whereas GPi responses similar to figure 3D are used to complete large amplitude movements.

\subsection{Dopamine Depletion Effects on the Discharge of Cells in the Primary Motor and Posterior Parietal Cortices}

Figure 4 depicts a composite schematic of simulated discharges of neurons in the primary motor and posterior parietal cortices in normal and dopamine depleted conditions. An increase in baseline activity of area's 4 bidirectional and reciprocal cells is evident as it has been observed experimentally [10]. Also, a significant reduction of peak activity of the bidirectional and reciprocal cells in the dopamine 
depleted case [10]. Finally, a disinhibition of reciprocally activated cells is observed in column 2 of figure 4D.

\subsection{Dopamine Depletion Effects on the Discharge of Cells in the Spinal Cord}

Figure 5 depicts a qualitative comparison of alpha-MN activity in normal and dopamine depleted conditions for small and large amplitude movements. In the dopamine depleted case, a significant decrease in the peak alpha-MN activity of both agonist and antagonist muscles is observed [20]. What is also evident is the absence of a co-contractive activation of antagonist muscles as it has been observed in monkey stimulation studies [21], but not in human studies [20].

A

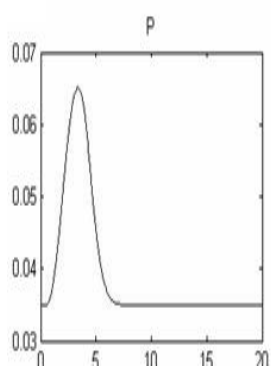

$\mathrm{C}$

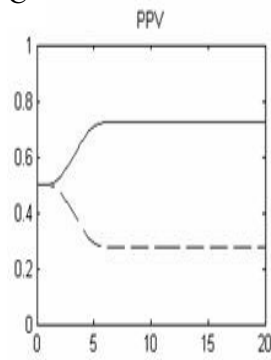

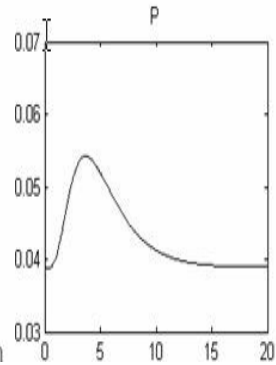

PPV

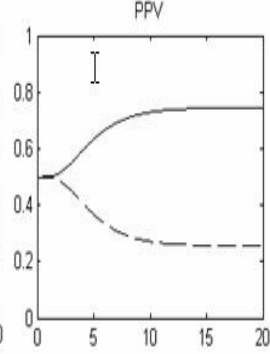

B
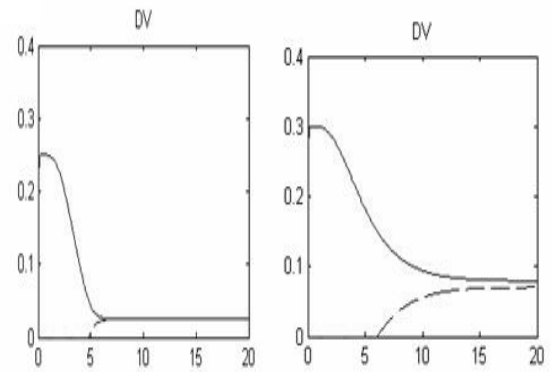

$\mathrm{D}$
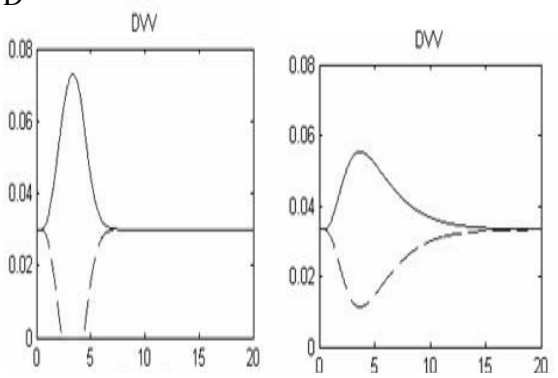

Fig. 4. Model dopamine depleted cell responses of primary motor and posterior parietal cortices in normal (column 1 of A, B, C, and D) and dopamine depleted (column 2 of A, B, C, and D) conditions. P: bidirectional neuronal response; DV: posterior area 5 phasic cell response; PPV: area 4 tonic cell response; DVV: area 4 reciprocal (phasic) cell response. Time (x-axis) in ms.

Also, a repetitive biphasic agonist-antagonist muscle activation can be observed as in [22]. The GO signal used to simulate such repetitive muscle activation is the one from figure $3 \mathrm{~B}$.

\subsection{Dopamine Depletion Effects on Movement Variables}

Figure 6 depicts the position, velocity and force profiles produced in the dopamine depleted condition of a large-amplitude movement. The GO signal used for these 
A

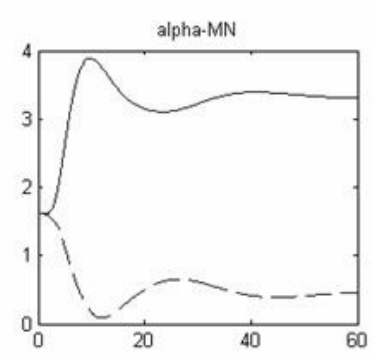

B

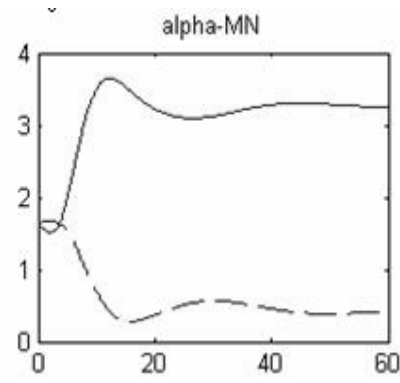

$\mathrm{C}$

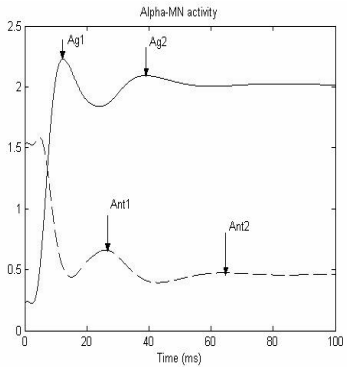

Fig. 5. Comparison of $(\mathrm{A})$ simulated triphasic alpha motorneuronal $(\mathrm{MN})$ activation under normal conditions, (B) simulated disrupted a-MN activation under dopamine depleted conditions, and (C) simulated repetitive biphasic a-MN activity in a dopamine depleted movement. The agonist a-MN activity is scaled down by 1.6 marks, so that a clearer repetitive biphasic pattern of muscle activation is shown. The GO signal used in (B) is the same as in figure $3 \mathrm{~B}$, whereas the $\mathrm{GO}$ signal used in $(\mathrm{C})$ is the same as in figure $6 \mathrm{~A}$. Time (x-axis) in ms.

A
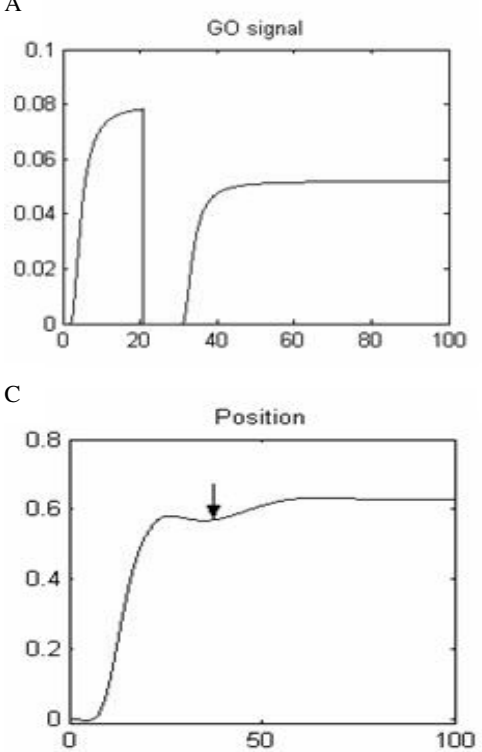

B

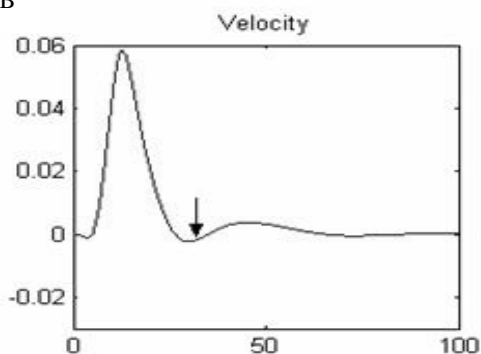

D

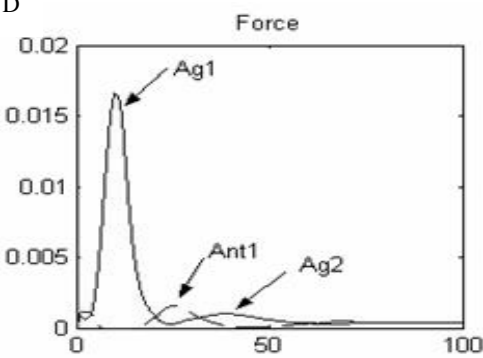

Fig. 6. Simulated repetitive GO signal (A), velocity (B), position (C) and muscle force (D) profiles in a large amplitude movement in dopamine-depleted condition. Down pointing arrows indicate sub-movements needed to complete the movement.

simulated is shown in figure 6A. They are evident the two sub-movements required to complete the movement. Figure 7 depicts the position, velocity and force profiles produced in the dopamine depleted condition of a small-amplitude movement. The GO signal used for these simulated is shown in figure 7A. For this movement a single motor command produced by the DVV cells is needed to complete it. 
A

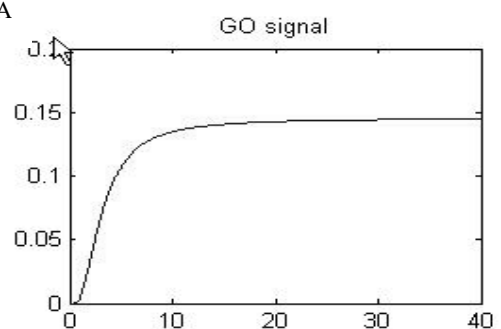

$\mathrm{C}$

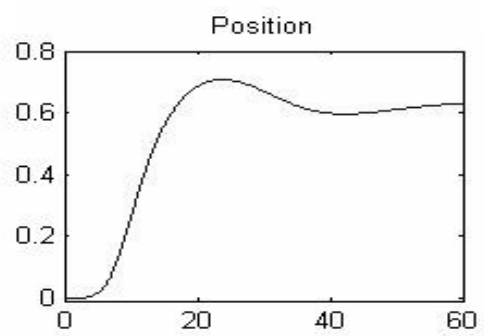

B

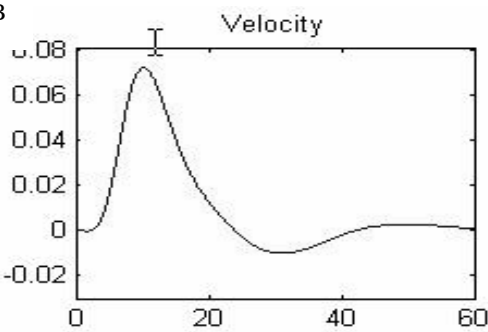

$\mathrm{D}$

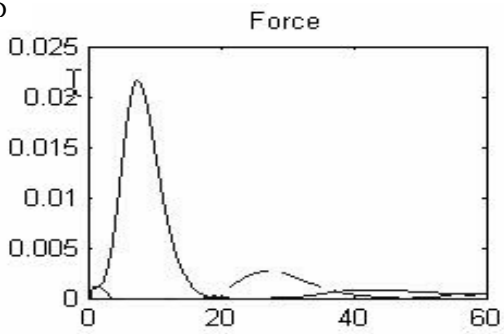

Fig. 7. Simulated GO signal (A), velocity (B), position (C) and muscle force (D) profiles in a small amplitude movement in dopamine-depleted condition

\section{Conclusion}

The present model is a model of voluntary movement and proprioception that offers an integrated interpretation of the functional roles of the diverse cell types in movement related areas of the primate cortex. The model is based on known corticospinal neuroanatomical connectivity (see Tables 1 and 2 of [8]). The model is successful at providing an integrative perspective on cortico-spinal control of parkinsonian voluntary movement by studying the effects of dopamine depletion on the output of the basal ganglia, cortex and spinal cord. It can account for the many known empirical signatures of Parkinsonian willful action such as

- Reduction of firing intensity and firing rate of cells in primary motor cortex Abnormal oscillatory GPi response

- Disinhibition of reciprocally tuned cells

- Repetitive bursts of muscle activation

- Reduction in the size and rate of development of the first agonist burst of EMG activity

- Asymmetric increase in the time-to-peak and deceleration time

- Decrease in the peak value of the velocity trace

- Increase in movement duration

- Substantial reduction in the size and rate of development of muscle production

These findings provide enough evidence to support the main hypothesis of the model reported earlier in the paper. A much larger set of experimental evidence that the model successfully simulated are shown in [12]. 


\section{References}

1. Albin, R. L., Young, A. B., \& Penney, J. B.: The functional anatomy of basal ganglia disorders. Trends in Neurosciences. 12 (1989) 366-375

2. Bullock, D., \& Contreras-Vidal, J. L.: How spinal neural networks reduce discrepancies between motor intention and motor realization. In K. Newel, \& D. Corcos (Eds.), Variability and motor control (pp. 183-221). Champaign, IL: Human Kinetics Press, 1993.

3. Bullock, D., Grossberg, S.: Neural dynamics of planned arm movements: Emergent invariants and speed-accuracy properties during trajectory formation. Psychological Review. 95 (1988) 49-90.

4. Bullock, D., Grossberg, S.: VITE and FLETE: Neural modules for trajectory formation and tension control. In W. Hershberger (Ed.), Volitional action (pp. 253-297). Amsterdam, The Netherlands: North-Holland, 1992.

5. Bullock, D., Grossberg, S.: Adaptive neural networks for control of movement trajectories invariant under speed and force rescaling. Human Movement Science. 10 (1991) 3-53.

6. Bullock, D., Grossberg, S.: Emergence of triphasic muscle activation from the nonlinear interactions of central and spinal neural networks circuits. Human Movement Science. 11 (1992) 157-167.

7. Bullock, D., Cisek, P., Grossberg, S.: Cortical networks for control of voluntary arm movements under variable force conditions. Cerebral Cortex. 8 (1998) 48-62.

8. Contreras-Vidal, J. L., Grossberg, S., Bullock, D.: A neural model of cerebellar learning for arm movement control: Cortico-spino-cerebellar dynamics. Learning and Memory. 3(6) (1997) 475-502.

9. Humphrey, D. R., \& Reed, D. J.: Separate cortical systems for control of joint movement and joint stiffness: Reciprocal activation and coactivation of antagonist muscles. In J. E. Desmedt (Ed.), Motor control mechanisms in health and disease. New York: Raven Press, 1983.

10. Doudet, D. J., Gross, C., Arluison, M., Bioulac, B.: Modifications of precentral cortex discharge and EMG activity in monkeys with MPTP induced lesions of DA nigral lesions. Experimental Brain Research. 80 (1990) 177-188.

11. Dormand, J. R., \& Prince, P. J.: A family of embedded Runge-Kutta formulae. Journal of Computational and Applied Mathematics. 6 (1980) 19-26.

12. Cutsuridis, V., Perantonis, S. (in press): A Neural Model of Parkinson's Disease Bradykinesia. Neural Networks.

13. Cutsuridis, V.: A neural network model of normal and Parkinsonian EMG activity of fast arm movements. Book of abstracts of the 18 Conference of Hellenic Society for Neuroscience, Athens, Greece, October 17-19, 2003.

14. Cutsuridis, V., Bullock, D.: A Neural Circuit Model of the Effects of Cortical Dopamine Depletion on Task-Related Discharge Patterns of Cells in the Primary Motor Cortex. Rethymnon, Crete, Book of abstracts of the $17^{\text {th }}$ Conference of Hellenic Society for Neuroscience, Poster 3, p. 39, October 4-6, 2002.

15. Cutsuridis, V., Bullock, D.: A Neural Circuit Model of the Effects of Cortical Dopamine Depletion on Task-Related Discharge Patterns of Cells in the Primary Motor Cortex. Poster Session II: Sensory-Motor Control and Robotics, Book of abstracts of the 6th International Neural Network Conference, Boston, MA, May 30 - June 1, 2002.

16. Stelmach, G.E., Teasdale, N., Phillips, J., Worringham, C.J.: Force production characteristics in Parkinson's disease. Exp Brain Res. 76 (1989) 165-172.

17. Rand, M.K., Stelmach, G.E., Bloedel, J.R.: Movement Accuracy Constraints in Parkinson's Disease Patients. Neuropsychologia. 38 (2000) 203-212. 
18. Camarata, P.J., Parker, P.G., Park, S.K., Haines, S.J., Turner, D.A., Chae, H., Ebner, T.J.: Effects of MPTP induced hemiparkinsonism on the kinematics of a two-dimensional, multi-joint arm movement in the rhesus monkey. Neuroscience. 48(3) (1992) 607-619.

19. Tremblay, L., Filion, M., \& Bedard, P. J.: Responses of pallidal neurons to striatal stimulation in monkeys with MPTP-induced parkinsonism. Brain Research. 498(1) (1989) 17-33.

20. Godaux, E., Koulischer, D., \& Jacquy, J.: Parkinsonian bradykinesia is due to depression in the rate of rise of muscle activity. Annals of Neurology. 31(1) (1992) 93-100.

21. Benazzouz, A., Gross, C., Dupont, J., Bioulac, B.: MPTP induced hemiparkinsonism in monkeys: Behavioral, mechanographic, electromyographic and immunohistochemical studies. Experimental Brain Research. 90 (1992) 116-120.

22. Hallett, M., Khoshbin, S.: A physiological mechanism of bradykinesia. Brain. 103 (1980) 301-314. 